\title{
Norm of Gaussian integers in arithmetical progressions and narrow sectors
}

\author{
S. Varbanets and Y. Vorobyov
}

Communicated by Yu. A. Drozd

\begin{abstract}
We proved the equidistribution of the Gaussian integer numbers in narrow sectors of the circle of radius $x^{\frac{1}{2}}, x \rightarrow \infty$, with the norms belonging to arithmetic progression $N(\alpha) \equiv \ell$ $(\bmod q)$ with the common difference of an arithmetic progression $q$, $q \ll x^{\frac{2}{3}-\varepsilon}$.
\end{abstract}

\section{Introduction}

For the classical arithmetic functions $\tau(n)$ (the number of divisors for the positive integer $n$ ) and $r(n)$ (the number of representations for the positive integer $n$ as sum of two squares of integers) there were obtained the asymptotic formulas of the sums

$$
\sum_{\substack{n \equiv(\bmod q) \\ n \leqslant x}} \tau(n) \text { and } \sum_{\substack{n \equiv \ell(\bmod q) \\ n \leqslant x}} r(n),
$$

where $q$ grows together with $x$ and they are nontrivial for $q \ll x^{\frac{2}{3}-\varepsilon}$.

For the function $\tau(n)$ K. Liu, I. Shparlinskii and T. Zhang ([2]) obtained the extended region of non-triviality.

In the present paper we investigate the distribution of points from complex plane $\mathbb{C}=\{x+i y \mid x, y \in \mathbb{R}\}, \varphi_{1}<\arg (x+i y) \leqslant \varphi_{2}, \varphi_{2}-\varphi_{1}<$

2010 MSC: 11L07, 11T23.

Key words and phrases: Gaussian integers, norm groups, Hecke $Z$-function, functional equation. 
$\frac{\pi}{2}, x^{2}+y^{2} \equiv \ell(\bmod q), x^{2}+y^{2} \leqslant N$. Using the property of Hecke $Z$ function of the quadratic field $\mathbb{Q}(i)$ and the estimates of special exponential sums, we obtain a non-trivial asymptotic formula for the number of integer points under the circle's sectorial region in arithmetic progression with the growing difference progression.

Throughout this paper we use the following notations.

- $p$ denotes a prime number in $\mathbb{Z}$;

- the Latin letters $a, b, k, m, n, \ell$ be the positive integers;

- $\Re z$ denotes the real part of $z$ and $\Im z$ be the imaginary part of $z$;

- through $\mathbb{Z}$ we denote the ring of integers;

- $G=\mathbb{Z}[i]$ denotes the ring of Gaussian integers $a+b i, a, b \in \mathbb{Z}$, $i^{2}=-1$;

- $G_{\gamma}$ (respectively, $G_{\gamma}^{*}$ ) be the ring of residue classes modulo $\gamma$ (respectively, the multiplicative group of inversive element in $G_{\gamma}$ );

- $N(\omega)$ is the norm of $\omega \in G, N(\omega)=|\omega|^{2}$;

- $S p(\omega)$ is the trace of $\omega$ from $\mathbb{Q}(i)$ to $\mathbb{Q}, S p(\omega)=2 \Re \omega$;

- symbols "«" and " $O$ " are equivalent;

- $s=\sigma+i t \in \mathbb{C}, \Re s=\sigma, \Im s=t$;

- $\chi_{q}$ denotes the Dirichlet character modulo $q$ over $\mathbb{Z}$

- $(a, q)=\operatorname{gcd}(a, q)$ in $\mathbb{Z}$;

- $(\alpha, \omega)=\operatorname{gcd}(\alpha, \omega)$ in $G$;

\section{Auxiliary results}

Let $\delta_{1}, \delta_{2} \in \mathbb{Q}(i)$ and $s=\sigma+i t$. For the rational integer number $m$ let us define the function sized by absolutely convergent series into semiplane $\Re s>1$ :

$$
Z_{m}\left(s, ; \delta_{1}, \delta_{2}\right):=\sum_{\omega \in G} \frac{e^{4 m i \arg \omega+\delta_{1}}}{N\left(\omega+\delta_{1}\right)^{s}} e^{\pi i S p\left(\delta_{2} \cdot \omega\right)} .
$$

It is obvious that with $m=0$ we get the Epstein zeta-function. With $\delta_{1}, \delta_{2} \in \mathbb{Q}_{i}$ we get the Hecke Z-function over the imaginary quadratic field $\mathbb{Q}(i)$.

Let $p>2$ be a prime rational number, $n \in \mathbb{N}$. Denote

$$
E_{p^{n}}:=\left\{\alpha \in G_{p^{n}} \mid N(\alpha) \equiv \pm 1 \quad\left(\bmod p^{n}\right)\right\} .
$$

It is also obvious that $E_{n}$ is the subgroup of multiplicative group of residue classes modulo $p^{n}$ over the $\operatorname{ring} G_{p^{n}}$.

We call $E_{p^{n}}$ the norm group in $G_{p^{n}}^{*}$. 
Lemma 1. Let $p \equiv 3(\bmod 4)$ and $E_{n}$ be the norm group in $G_{p^{n}}$. Then $E_{n}$ is the cyclic group, $\left|E_{n}\right|=2(p+1) p^{n-1}$, and let $u+i v$ be a generative element of $E_{n}$. Then exist $x_{0}, y_{0} \in \mathbb{Z}_{p^{n}}^{*}$ such that

$$
\begin{gathered}
(u+i v)^{2(p+1)} \equiv 1+p^{2} x_{0}+i p y_{0}, \\
2 x_{0}+y_{0}^{2} \equiv-2 p^{2} x_{0}^{2} \quad\left(\bmod p^{3}\right) .
\end{gathered}
$$

Moreover, we have modulo $p^{n}$ for any $t=4,5, \ldots, p^{n-1}-1$,

$$
\begin{aligned}
& \Re\left((u+i v)^{2(p+1) t}\right)=A_{0}+A_{1} t+A_{2} t^{2}+\cdots \\
& \Im\left((u+i v)^{2(p+1) t}\right)=B_{0}+B_{1} t+B_{2} t^{2}+\cdots,
\end{aligned}
$$

where

$$
\begin{gathered}
A_{0} \equiv 1 \quad\left(\bmod p^{4}\right), \quad B_{0} \equiv 0 \quad\left(\bmod p^{4}\right), \\
A_{1} \equiv p^{2} x_{0}+\frac{1}{2} p^{2} y_{0}^{2} \equiv-\frac{5}{2} x_{0}^{2} p^{4} \quad\left(\bmod p^{5}\right), \\
B_{1} \equiv p y_{0}\left(1-p^{2} x_{0}\right) \quad\left(\bmod p^{4}\right), \\
A_{2} \equiv-\frac{5}{2} x_{0}^{2} p^{2} \quad\left(\bmod p^{5}\right), \quad B_{2} \equiv \frac{5}{3} p^{3} x_{0} y_{0} \quad\left(\bmod p^{4}\right), \\
A_{j} \equiv B_{j} \equiv 0 \quad\left(\bmod p^{3}\right), \quad j=3,4, \ldots
\end{gathered}
$$

(In greater details see [3])

Denote

$$
\begin{gathered}
(u+i v)^{k}=u(k)+i v(k), \quad 0 \leqslant k \leqslant 2 p+1, \\
(u+i v)^{2(p+1) t+k} \equiv \sum_{j=0}^{n-1}\left(A_{j}(k)+i B_{j}(k)\right) t^{k} \quad\left(\bmod p^{n}\right) .
\end{gathered}
$$

It is obvious that

$$
A_{j}(k)=A_{j} u(k)-B_{j} v(k), \quad B_{j}(k)=A_{j} v(k)+B_{j} u(k) .
$$

Thus from Lemma 1 we infer

Corollary. For $k=0,1, \ldots, 2 p+1$ we have

$$
\begin{gathered}
u(0)=1, \quad v(0)=0, \quad(u(p+1), p)=1, \quad p \| v(p+1) ; \\
(u(k), p)=(v(k), p)=1 \quad \text { for } \quad k \neq 0 \quad\left(\bmod \frac{p+1}{2}\right) ; \\
u(k) \equiv 0 \quad(\bmod p), \quad(v(k), p)=1 \quad \text { if } \quad k=\frac{p+1}{2} \quad \text { or } \quad \frac{3 p+1}{2} ; \\
u(k) \equiv u(-k), \quad v(k) \equiv-v(-k) .
\end{gathered}
$$


Hence, for $k \not \equiv 0\left(\bmod \frac{p+1}{2}\right)$ we have

$$
\begin{gathered}
A_{0}(k) \equiv u(k) \quad(\bmod p), \quad B_{0}(k) \equiv v(k) \quad(\bmod p), \\
A_{1}(k) \equiv-p y_{0} v(k), \quad B_{1}(k) \equiv p y_{0} u(k) \quad\left(\bmod p^{2}\right) \\
A_{2}(k) \equiv-\frac{5}{2} x_{0}^{2} p^{2} u(k), \quad B_{2}(k) \equiv-\frac{5}{2} x_{0}^{2} p^{2} v(k) \quad\left(\bmod p^{4}\right) .
\end{gathered}
$$

For $k=\frac{p+1}{2}$ or $\frac{3 p+1}{2}$ we obtain

$$
p\left\|A_{1}(k), \quad p^{2}\right\| B_{1}(k), \quad p^{2} \| A_{2}(k), \quad B_{2}(k) \equiv 0 \quad\left(\bmod p^{3}\right) .
$$

Moreover,

$$
\begin{gathered}
A_{1}(0) \equiv-\frac{5}{2} x_{0}^{2} p^{4} \quad\left(\bmod p^{5}\right), \quad B_{1}(0) \equiv 0 \quad\left(\bmod p^{4}\right) \\
A_{2}(0) \equiv-\frac{5}{2} x_{0}^{2} p^{2} \quad\left(\bmod p^{5}\right), \quad B_{2}(0) \equiv 0 \quad\left(\bmod p^{3}\right), \quad p^{2} \| A_{1}(p+1), \\
p\left\|B_{1}(p+1), \quad p^{2}\right\| A_{2}(p+1), \quad B_{2}(p+1) \equiv 0 \quad\left(\bmod p^{3}\right) .
\end{gathered}
$$

At last for all $k=0,1, \ldots, 2 p+1$

$$
A_{j}(k) \equiv B_{j}(k) \equiv 0 \quad\left(\bmod p^{3}\right), \quad j=3,4, \ldots
$$

Lemma 2. Let $q=p^{\ell}$ with $\ell \geqslant 1, g(y)$ is the polynomial in form

$$
g(y)=A_{1} y+p A_{2} y^{2}+p^{\lambda_{3}} A_{3} y^{3}+\cdots+p^{\lambda_{k}} A_{k} y^{k}, \quad k \geqslant 3,
$$

with $A_{j} \in \mathbb{Z},\left(A_{j}, p\right)=1, j=3, \ldots, k, 2 \leqslant \lambda_{3} \leqslant \lambda_{4} \leqslant \cdots \leqslant \lambda_{k}$. Then we have

$$
S_{q}:=\sum_{y=1}^{q-1} e^{2 \pi i \frac{g(y)}{p^{\ell}}}=p^{\left[\frac{\ell}{2}\right]} \sum_{\substack{y \in \mathbb{Z}_{p^{[\ell / 2]}} \\ g^{\prime}(y) \equiv 0\left(\bmod p^{[\ell / 2]}\right)}} B_{q}(y),
$$

where

$$
B_{q}(y)=\left\{\begin{array}{lll}
0 & \text { if }\left(A_{1}, p\right)=1, \\
1 & \text { if } \ell \equiv 0 \quad(\bmod 2), \\
& A_{1} \equiv 0 \quad(\bmod p), \\
\sum_{z=0}^{p-1} e^{2 \pi i \frac{\left(\left(\frac{A_{1}}{p}+2 A_{2}\right) z+2 z^{2}\right)}{p}} & \text { if } \ell \equiv 1 \quad(\bmod 2), \\
& & A_{1} \equiv 0 \quad(\bmod p) .
\end{array}\right.
$$

Proof. The proof of this assertion repeats the proofs of Lemmas 12.3 and 12.4 in [1]. 
For $p \equiv 1(\bmod 4)$ or $p=2$ the norm groups are not the cyclic groups. We shall use the description of the solutions $x^{2}+y^{2} \equiv 1\left(\bmod p^{n}\right)$ for these cases.

Lemma 3. Let $(x, y)$ is a solution of the congruence $x^{2}+y^{2} \equiv 1\left(\bmod p^{\ell}\right)$, $p>2$ is a prime number. Then all solutions with $\left(x_{0}, p\right)=1$ are described in the following manner

$$
x=x(0) f\left(y_{0}, t\right), \quad y=y_{0}+p t, \quad t=0,1, \ldots, p^{\ell-1}-1,
$$

where $x(0)$ runs all solutions of the congruence

$$
x^{2} \equiv 1-y_{0}^{2} \quad\left(\bmod p^{n}\right),
$$

$y_{0}$ runs all solutions of the congruence

$$
x_{0}^{2}+y_{0}^{2} \equiv 1 \quad(\bmod p)
$$

with $x_{0} \not \equiv 0(\bmod p)$, and

$f\left(y_{0}, t\right)=1+p \frac{y_{0}}{y_{0}^{2}-1} t+p^{2} \frac{1-y_{0}}{y_{0}^{2}-1} t^{2}+p^{\lambda_{3}} X_{3}\left(y_{0}\right) t^{3}+\cdots+p^{\lambda_{s}} X_{s}\left(y_{0}\right) t^{s}$,

under conditions $\left(X_{j}\left(y_{0}\right), p\right)=1, \lambda_{j} \geqslant 3, s \leqslant\left[\ell \frac{p-1}{p-2}\right]$.

For the solutions of the congruence $x^{2}+y^{2} \equiv 1\left(\bmod p^{\ell}\right)$ with $x_{0} \equiv 0$ $(\bmod p)$ we have

$$
x=p t, \quad y \equiv \pm\left(1-\frac{1}{2} p^{2} t^{2}\right) \quad\left(\bmod p^{4}\right) .
$$

(Here, the multiplicative inverse for 2 and $y_{0}^{2}-1$ is considered modulo $p^{n}$ ).

Lemma $3^{\prime}$. Let $s=\left[\frac{\ell-1}{2}\right]$. There exists the polynomial

$$
f(t)=1+2^{\lambda_{1}} A-1 t^{2}+\cdots+2^{\lambda_{s}} A_{s} t^{2 s},
$$

with $A_{j} \equiv 1(\bmod 2), \lambda_{j} \geqslant 2 j+1, j=1, \ldots, s$, such that all solutions of the congruence $x^{2}+y^{2} \equiv 1\left(\bmod p^{\ell}\right)$ can be written as

$$
\begin{aligned}
& x=4 t, \quad y= \pm f(t) \quad \text { or } \quad x=4 t, \quad y= \pm\left(2^{\ell-1}-1\right) f(t), \\
& t=0,1, \ldots, 2^{\ell-2}-1 .
\end{aligned}
$$


Lemma 4. Let us $I(\ell, q)$ be the number of solutions of the congruence

$$
u^{2}+v^{2} \equiv a \quad(\bmod q), \quad(a, q)=\prod_{p \mid q} p^{t_{0}}
$$

Then we have

$$
\begin{aligned}
& I(a, q) \\
& =c(a, q) q \prod_{p^{t} \| q}\left(1-\frac{\chi_{4}\left(p^{t_{0}+1}\right)}{p}\left(1-\chi_{4}\left(p^{t-t_{0}}\right)\right)+\left(1-\frac{1}{p}\right) \sum_{b=t-t_{0}}^{t-1} \chi_{4}\left(p^{t-b}\right)\right),
\end{aligned}
$$

where

$$
c(a, q)= \begin{cases}1 & \text { if }(q, 2)=1, \\ 1 & \text { if } 2 \| q, \\ 1 & \text { if } q \equiv 0 \quad(\bmod 4), t_{0}>t-2, \\ 2 & \text { if } q \equiv 0 \quad(\bmod 4), t_{0}<t-2 \text { and } \frac{a}{2^{t_{0}}} \equiv 1 \quad(\bmod 4), \\ 0 & \text { if } q \equiv 0 \quad(\bmod 4), t_{0} \leqslant t-2 \text { and } \frac{a}{2^{t_{0}}} \equiv 3 \quad(\bmod 4)\end{cases}
$$

This lemma follows from the equation

$$
I\left(a, p^{t}\right)=\sum_{u, v \in \mathbb{Z}_{p^{t}}} \frac{1}{p^{t}} \sum_{z \in \mathbb{Z}_{p^{t}}^{*}} e^{2 \pi i \frac{z\left(y^{2}+v^{2}-\ell\right)}{p^{t}}}
$$

and the values of the Gaussian sums $\sum_{x \in \mathbb{Z}_{p} t} e^{2 \pi i \frac{z x^{2}}{p^{t}}}$.

Similarly, we obtain the description of the solutions of the congruence $x^{2}+y^{2} \equiv-1\left(\bmod p^{\ell}\right), p \equiv 1(\bmod 4)$. Indeed, let $c_{0}$ be the solution of the congruence $x^{2} \equiv-1\left(\bmod p^{\ell}\right)$. Then

$$
x=c_{0} x(0) f_{1}\left(y_{0}, t\right), \quad y=y_{0}+p t, \quad t=0,1, \ldots, p^{\ell-1}-1,
$$

where $f_{1}\left(y_{0}, t\right)$ is as $f\left(y_{0}, t\right)$.

\section{The main results}

We consider the generalized Hecke $Z$-function of quadratic field $\mathbb{Q}(i)$

$$
Z_{m}\left(s ; \delta_{1}, \delta_{2}\right):=\sum_{\substack{\omega \in G \\ \omega \neq \delta_{1}}} \frac{e^{4 m i \arg \left(\omega+\delta_{1}\right)}}{N\left(\omega+\delta_{1}\right)} e^{\pi i S p\left(\omega \delta_{2}\right)}, \quad(\Re s>1),
$$


where $\delta_{1}, \delta_{2} \in \mathbb{Q}(i), m \in \mathbb{Z}$. This function satisfies the functional equation

$$
\begin{aligned}
& \pi^{-1} \Gamma(2|m|+s) Z_{m}\left(s ; \delta_{1}, \delta_{2}\right) \\
& \quad=\pi^{-(1-s)} \Gamma(2|m|+1-s) Z_{-m}\left(1-s ;-\delta_{2}, \delta_{1}\right) e^{\pi i S p\left(\delta_{1} \delta_{2}\right)} .
\end{aligned}
$$

The function $Z_{m}\left(s ; \delta_{1}, \delta_{2}\right)$ is an entire function except the case $m=0$ and the Gaussian integer $\delta_{2}$ when $Z_{m}\left(s ; \delta_{1}, \delta_{2}\right)$ is holomorphic for all complex $s$ exclusive $s=1$ where it has a simple pole with residue $\pi$.

We define the multiplicative character modulo $q$ over $G_{p^{\ell}}^{*}$ as

$$
\chi(\omega)=\chi_{p^{\ell}}(N(\omega))
$$

where $\chi_{p^{\ell}}$ is the character modulo $p^{\ell}$ in $\mathbb{Z}_{p^{*}}^{*}$.

Let $\Xi_{m}(\omega):=e^{4 m i \arg \omega} \chi(\omega)=e^{4 m i \arg \omega} \chi_{p^{\ell}}(N(\omega))$. Then from (9) we have for $Z\left(s ; \Xi_{m}\right):=\sum_{\omega} \frac{\Xi_{m}(\omega)}{N(\omega)^{s}}$ the following functional equation

$$
Z\left(s ; \Xi_{m}\right)=\kappa\left(\Xi_{m}\right) \Psi\left(s, \Xi_{m}\right) Z\left(1-s, \bar{\Xi}_{m}\right),
$$

where

$$
\begin{aligned}
& \kappa\left(\Xi_{m}\right)=\left(N\left(p^{\ell}\right)\right)^{-\frac{1}{2}} \sum_{\tau \in G_{p^{\ell}}} \chi(N(\tau)) e^{S p \frac{\tau}{p^{\ell}}}, \\
& \Psi\left(s, \Xi_{m}\right)=\left(\frac{1}{\pi} N\left(p^{\ell}\right)^{\frac{1}{2}}\right)^{1-2 s} \frac{\Gamma(2|m|+1-s)}{\Gamma(2|m|+s)} .
\end{aligned}
$$

Denote

$$
r_{m}(n)=\sum_{\substack{u, v \in \mathbb{Z} \\ u^{2}+v^{2}=n}} e^{4 m i \arg (u+i v)} .
$$

From this we have

$$
\sum_{n \leqslant x} r_{m}(n) \chi_{p^{\ell}}(n)=\sum_{\substack{u, v \in \mathbb{Z} \\ u^{2}+v^{2}=n \leqslant x}} e^{4 m i \arg (u+i v)} \chi_{p^{\ell}}(n) .
$$

Therefore,

$$
F_{m}(s)=\sum_{n=1}^{\infty} \frac{r_{m}(n)}{n^{s}}=\sum_{\chi_{q}} \bar{\chi}_{q}(a) \cdot Z\left(s ; \Xi_{m}\right) .
$$


We get by the Perron's formula on an arithmetic progression with $c>1$, $T>1,\left(a, p^{\ell}\right)=1,0<\varepsilon<\frac{1}{2}$ the following equality

$$
\begin{aligned}
& \sum_{n \equiv a\left(\underset{n \leqslant x}{\left.\bmod p^{\ell}\right)}\right.} r_{m}(n)=\frac{1}{2 \pi i} \int_{c-i T}^{c+i T} F_{m}(s) \frac{x^{s}}{s} d s+O\left(\frac{x^{c}}{T p^{\ell}(c-1)}\right)+O\left(x^{\varepsilon}\right) \\
& \quad=\operatorname{res}_{s=0,1}\left(F_{m}(s) \frac{x^{s}}{s}\right)+\frac{1}{2 \pi i} \int_{-\varepsilon-i T}^{-\varepsilon+i T} F_{m}(s) \frac{x^{s}}{s} d s+\max _{-\varepsilon \leqslant \Re s \leqslant c}\left|\frac{1}{s} F_{m}(s) x^{s}\right| \\
& \quad+O\left(\frac{x^{c}}{T p^{\ell}(c-1)}\right)+O\left(x^{\varepsilon}\right),
\end{aligned}
$$

where $\varepsilon$ is a positive arbitrary small number.

From the functional equation for $Z(s, \Xi)$, summing all over character $\chi_{p^{\ell}}$, we have for $\Re s<0$

$$
F_{m}(s)=\pi^{-1+2 s} \frac{\Gamma(2|m|+1-s)}{\Gamma(2|m|+s)} \times \sum_{\substack{\omega \in G \\\left(\omega, p^{\ell}\right)=1}} \frac{e^{-4 m i \arg \omega}}{N(\omega)^{1-s}} \sum_{\substack{\tau \in G_{p^{\ell}}^{*} \\ N(\tau) \equiv a N(\omega)\left(\bmod p^{\ell}\right)}} e^{\frac{s p(\tau)}{p^{\ell}}} .
$$

Consider the sum

$$
\sum_{0}:=\sum_{\substack{\tau \in G_{p^{\ell}}^{*} \\ N(\tau) \equiv n\left(\bmod p^{\ell}\right) \\\left(n, p^{\ell}\right)=1}} e^{\pi i S p\left(\frac{\tau}{p^{\ell}}\right)} .
$$

For $p \equiv 3(\bmod 4)$, we apply the representation of elements from the norm group $E_{p^{\ell}}$. Lemma 1 and its Corollary give

$$
\begin{aligned}
\sum_{0} & =\sum_{k=0}^{2 p+1} e^{2 \pi i \frac{A_{0}^{\prime}(k)}{p^{\ell}}} \sum_{t=0}^{p^{\ell-1}-1} e^{2 \pi i \frac{A_{1}^{\prime}(k) t+A_{2}^{\prime}(k) t^{2}+\cdots}{p^{\ell}}} \\
& =e^{2 \pi i \frac{A_{0}^{\prime}(0)}{p^{\ell}}} \sum_{t=0}^{p^{\ell-1}-1} e^{2 \pi i \frac{A_{1}^{\prime}(0) t+A_{2}^{\prime}(0) t^{2}+\cdots}{p^{\ell}}} \\
& +e^{2 \pi i \frac{A_{0}^{\prime}(p+1)}{p^{\ell}}} \sum_{t=0}^{p^{\ell-1}-1} e^{2 \pi i \frac{A_{1}^{\prime}(p+1) t+A_{2}^{\prime}(p+1) t^{2}+\cdots}{p^{\ell}}},
\end{aligned}
$$

where $A_{j}^{\prime}(0)$ and $A_{j}^{\prime}(p+1)$ differ from $A_{j}(j)$ and $A-j(p+1)$ only by the multiplier $N(\omega) a$. 
Now Lemma 3 gives

$$
\begin{aligned}
E_{0}=p^{\frac{\ell}{2}} & \left(e^{2 \pi i \frac{A_{0}^{\prime}(0)}{p^{\ell}}}+e^{2 \pi i \frac{A_{0}^{\prime}(p+1)}{p^{\ell}}}\right) \\
& \times\left\{\begin{array}{lll}
1 & \text { if } \ell \equiv 0 & (\bmod 2), \\
e^{-\frac{2 \pi i A_{1}^{\prime}\left(2 A_{2}^{\prime}\right)^{-1}}{p}} & \text { if } \ell \equiv 1 & (\bmod 2) .
\end{array}\right.
\end{aligned}
$$

If $p \equiv 1(\bmod 4)$ or $p=2$ we use Lemma 1 and then obtain $E_{0}=$ $O\left(p^{\ell \frac{1}{2}}\right)$ with an absolute constant in the symbol "O".

Now we able to prove the main theorems.

Let us denote through $A\left(x ; \varphi_{1}, \varphi_{2} ; a, p^{\ell}\right)$ the number of points $(u, v)$ in the circle $\left(u^{2}+v^{2}\right) \leqslant x$ under conditions

$$
\begin{aligned}
& u, v \in \mathbb{Z}, \quad \varphi_{1}<\arg (u+i v) \leqslant \varphi_{2}, \\
& u^{2}+v^{2} \equiv a \quad\left(\bmod p^{\ell}\right), \quad\left(a, p^{\ell}\right)=1 .
\end{aligned}
$$

Theorem 1. For $x \rightarrow \infty$ the following estimate

$$
\begin{aligned}
\sum_{\substack{n \equiv a\left(\bmod p^{\ell}\right) \\
n \leqslant x}} r_{m}(n)=\varepsilon & \frac{\pi x}{p^{\ell}} k_{0}\left(1-\frac{\chi_{4}(p)}{p}\right) \\
& +O\left(\frac{x^{\frac{1}{2}+\varepsilon}}{p^{\frac{\ell}{4}}} M^{1+\varepsilon}\right)+O\left(p^{\frac{\ell}{2}} M^{1+\varepsilon}\right),
\end{aligned}
$$

holds, where $\varepsilon_{m}=0$ if $m \neq 0, \varepsilon_{0}=1, k_{0}=1$ if $p>2$, or $k=2$ if $p=2$, $\ell \geqslant 3 ; M=|m|+3, \varepsilon>0$ is an arbitrary small number; constants in the symbols can depend only on $\varepsilon$.

Proof. The function $F_{m}(s)$ has a pole in $s=1$ only if $m=0$ :

$$
\underset{s=1}{\operatorname{res}} F_{0}(s)=\frac{\pi x}{p^{\ell}} k_{0}\left(1-\frac{\chi_{4}(p)}{p}\right) .
$$

The estimate for $F_{m}(0)$ is easy proving by the Phragmen-Lindelöff principle and the estimates of $Z_{m}(s)$ on the bounds of stripe $-\varepsilon \leqslant \Re s \leqslant 1+\varepsilon$. Therefore, we have

$$
\underset{s=0}{\operatorname{res}} F_{m}(s) \ll p^{\frac{\ell}{2}}(|m|+3) \log (|m|+3) .
$$


Hence,

$$
\begin{aligned}
\sum_{\substack{n \equiv a\left(\bmod p^{\ell}\right) \\
n \leqslant x}} r_{m}(n)=\varepsilon_{m} \frac{\pi x}{p^{\ell}} \sum_{\substack{u, v \in \mathbb{Z}_{p^{\ell}} \\
u^{2}+v^{2} \equiv a\left(\bmod p^{\ell}\right)}} 1+O\left(p^{\left.\frac{\ell}{2}(|m|+3) \log (|m|+3)\right)+}\right. \\
+\frac{1}{2 \pi i} \int_{-\varepsilon-i T}^{-\varepsilon+i T} F_{m}(s) \frac{x^{s}}{s} d s+O\left(\frac{x^{c}}{T p^{\ell}(c-1)}+x^{\varepsilon}\right) .
\end{aligned}
$$

Note that

$$
\varepsilon_{m} \frac{\pi x}{p^{\ell}} \sum_{\substack{u, v \in \mathbb{Z}_{p}^{\ell} \\ u^{2}+v^{2} \equiv a\left(\bmod p^{\ell}\right)}} 1=\varepsilon_{m} \frac{\pi x}{p^{\ell}} k_{0}\left(1-\frac{\chi_{4}(p)}{p}\right),
$$

where

$$
F_{m}(s)=\pi^{-1+2 s} \frac{\Gamma(2|m|+1-s)}{\Gamma(2|m|+s)} \times \sum_{\substack{\omega \in G \\\left(\omega, p^{\ell}\right)=1}} \frac{e^{-4 m i \arg \omega}}{N(\omega)^{1-s}} \sum_{\substack{\tau \in G_{p^{\ell}}^{*} \\ N(\tau) \equiv a N(\omega)\left(\bmod p^{\ell}\right)}} e^{\pi i \frac{S p(\tau)}{p^{\ell}}} .
$$

Thus, using the estimate of the sum $\sum_{0}$ and the Stirling formula for the gamma-function $\Gamma(z)$, we at once obtain the estimate of the integral in $(16)$

$$
\frac{1}{2 \pi i} \int_{-\varepsilon-i T}^{-\varepsilon+i T} F_{m}(s) \frac{x^{s}}{s} d s \ll T^{1+2 \varepsilon} p^{\frac{\ell}{2}+\varepsilon} x^{-\varepsilon} \ll T^{1+2 \varepsilon} p^{\frac{\ell}{2}} .
$$

Choosing $c=1+(\log x)^{-1}, T=\frac{x^{\frac{1}{2}}}{p^{\frac{3 \ell}{4}}}$, we get assertion of Theorem 1 .

The following theorems stem from this result and Vinogradov's lemma (see, [4], Lemma 12, pp. 261-262).

Theorem 2. In the sectorial region $u^{2}+v^{2} \leqslant x, u^{2}+v^{2} \equiv a\left(\bmod p^{\ell}\right)$, $\varphi_{1}<\arg (u+i v) \leqslant \varphi_{2}, \varphi_{2}-\varphi_{1} \gg x$ the following asymptotic formula holds:

$$
\begin{aligned}
A\left(x ; \varphi_{1}, \varphi_{2} ; a, p^{\ell}\right):= & \sum_{\substack{u, v \\
u^{2}+v^{2} \equiv a\left(\bmod p^{\ell}\right) \\
\varphi_{1}<\arg (u+i v) \leqslant \varphi_{2} \\
u^{2}+v^{2} \leqslant x}} 1= \\
= & \frac{\varphi_{2}-\varphi_{1}}{2} \cdot \frac{k_{0} x}{p^{\ell}}\left(1-\frac{\chi_{4}(p)}{p}\right)+O\left(\frac{x^{\frac{1}{2}+\varepsilon}}{p^{\frac{\ell}{4}}}\right)
\end{aligned}
$$


Theorem 3. Let $p$ be a prime number, $\ell \geqslant 3$, and $p^{\frac{3 \ell}{2-4 \kappa}} \leqslant x \leqslant p^{2 \ell}$, $0<\kappa \leqslant \frac{1}{8}-\frac{1}{4 \ell}, \varphi_{2}-\varphi_{1} \gg x^{-\kappa}$. Then we have

$$
A\left(x ; \varphi_{1}, \varphi_{2} ; a, p^{\ell}\right)=\frac{\varphi_{2}-\varphi_{1}}{2} \cdot \frac{x}{p^{\ell}}\left(1-\frac{\chi_{4}(p)}{p}\right)+O\left(\frac{x^{1-\kappa}}{p^{\ell}} \log x^{\kappa}\right) .
$$

Actually, in Vinogradov's lemma we take $\Omega=\frac{\pi}{2}, \delta=x^{\kappa}, \Delta=x^{-\alpha}$ and let $\Delta \leqslant \varphi_{2}-\varphi_{1}<\frac{\pi}{4}-2 \kappa$. Then $f\left(\varphi_{1}, \varphi_{2}\right)$ be the function from that lemma.

Consider the function

$$
\Phi\left(\varphi_{1}, \varphi_{2}\right)=\frac{1}{4} \sum_{\substack{u^{2}+v^{2} \leqslant x \\ u^{2}+v^{2} \equiv a\left(\bmod p^{\ell}\right)}} f(\arg (u+i v)) .
$$

Then we have

$$
\begin{aligned}
\Phi\left(\varphi_{1}, \varphi_{2}\right) & =\sum_{\substack{u^{2}+v^{2} \leqslant x \\
u^{2}+v^{2} \equiv a\left(\bmod p^{\ell}\right)}} \sum_{m=-\infty}^{\infty} a_{m} e^{4 m i \arg (u+i v)}= \\
& =\sum_{m=-\infty}^{\infty} a_{m} \sum_{n \equiv a\left(\bmod p^{\ell}\right)} r_{m}(n),
\end{aligned}
$$

(here $a_{m}$ are the coefficients from the Vinogradov's lemma).

We take $r=3$ (in the notation of the Vinogradov's lemma) and take into account that

$$
\begin{aligned}
a_{0} & =\frac{1}{\Omega}\left(\varphi_{2}-\varphi_{1}+\Delta\right) \\
\left|a_{m}\right| & \leqslant\left\{\begin{array}{l}
\frac{1}{\Omega}\left(\varphi_{2}-\varphi_{1}+\Delta\right) \\
\frac{2}{\pi|m|} \\
\frac{2}{\pi|m|}\left(\frac{r \Omega}{\pi|m| \Delta}\right)^{r}
\end{array}\right.
\end{aligned}
$$

then after simple calculations we get Theorem 2 and Theorem 3 .

Taking into account that Hecke characters and Gauss exponential sums have the multiplicative properties modulo $q$, we have the following assertion. 
Theorem 4. In the sectorial region $u^{2}+v^{2} \leqslant x, u^{2}+v^{2} \equiv a(\bmod q)$, $\varphi_{1}<\arg (u+i v) \leqslant \varphi_{2}, \varphi_{2}-\varphi_{1} \gg x$ the following asymptotic formula holds:

$$
\begin{aligned}
A\left(x ; \varphi_{1}, \varphi_{2} ; a, q\right):= & \sum_{\substack{u, v \\
u^{2}+v^{2} \equiv a(\bmod q) \\
\varphi_{1}<\arg (u+i v) \leqslant \varphi_{2} \\
u^{2}+v^{2} \leqslant x}} 1= \\
= & \frac{\varphi_{2}-\varphi_{1}}{2} \cdot \frac{k_{0} x}{q} \prod_{p \mid q}\left(1-\frac{\chi_{4}(p)}{p}\right)+O\left(\frac{x^{\frac{1}{2}+\varepsilon}}{q^{\frac{1}{4}}}\right) .
\end{aligned}
$$

Remark. The result of Theorem 1 can be improved in case $p \equiv 3(\bmod 4)$ and $\ell \geqslant 3$ in view of the fact that we have the precise meaning of the sum $E_{0}($ see $(13))$.

\section{References}

[1] Iwaniec H., Kowalski E., Analytic Number Theory, Providence: American Mathematical Society. Colloquium Publications, vol. 53, 2004.

[2] Liu K., Shparlinski I.E., Zhang T., Divisor problem in arithmetic progressions modulo a prime power, Advances in Mathematics, 325(5), 2018, pp. 459-481.

[3] Varbanets S., Exponential sums on the sequences of inversive congruential pseudorandom numbers, Siauliai Math. Semin., 3(11), 2008, pp. 247-261.

[4] Vinogradov I.M., Izbrannye trudy. (Russian) [Selected works.], Izdat. Akad. Nauk SSSR, Moscow, 1952.

\section{CONTACT INFORMATION}

Sergey Varbanets Odessa I.I. Mechnikov National University, Dvoryanskaya str. 2, 65026 Odessa, Ukraine E-Mail(s): varb@sana.od.ua

Yakov Vorobyov

Izmail State Humanities University, Izmail, Repina str. 12, 68610 Izmail, Ukraine E-Mail(s): yashavo@mail.ru

Received by the editors: 20.01.2020. 